0

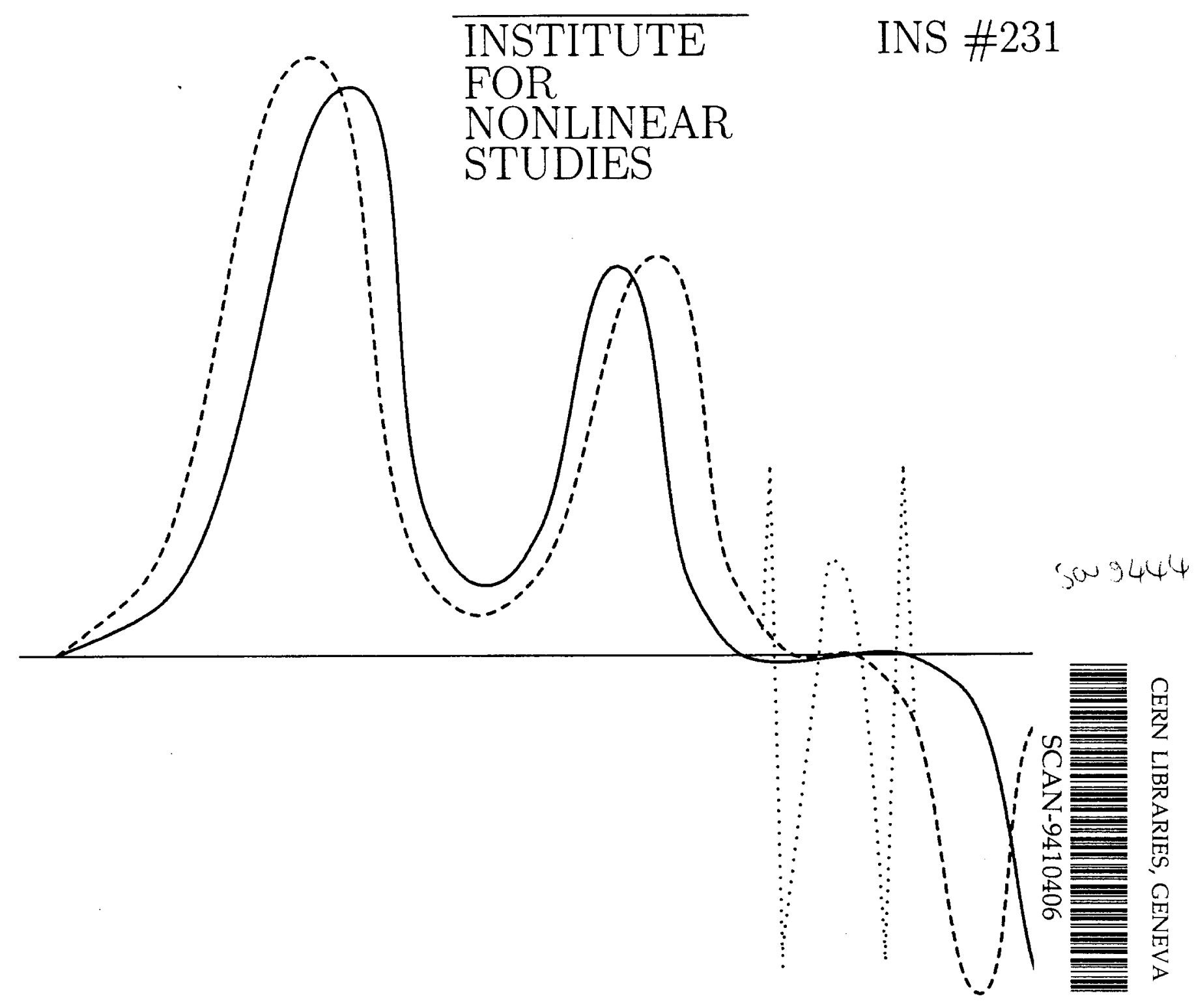

Generalized Conditional Symmetries and Exact Solutions of Non-Integrable Equations

by

A.S. Fokas and Q.M. Liu May 1993

CLARKSON UNIVERSITY

Potsdam, New York 13699, U.S.A. 



\title{
Generalized Conditional Symmetries and Exact Solutions of Non-Integrable Equations
}

\author{
A.S. Fokas and Q.M. Liu \\ Department of Mathematics and Computer Science \\ and Institute for Nonlinear Studies \\ Clarkson University \\ Potsdam, New York 13699-5815, U.S.A.
}

May 1993

INS \#231

\begin{abstract}
We introduce the concept of a generalized conditional symmetry. This concept provides an algorithm for constructing physically important exact solutions of non-integrable equations. Examples include 2-shock and 2-soliton solutions. The existence of such exact solutions for non-integrable equations can be traced back to the relation of these equations with integrable ones. In this sense these exact solutions are remnants of integrability.
\end{abstract}




\section{Introduction and Main Results}

It is well known that there exist nonlinear diffusion equations which possess exact traveling wave solutions. For example, the Fisher type equation $u_{t}=u_{x x}+u(1-u)$, admits an exact solution describing a wave traveling with speed $\pm 5[1]$. Another example is the Fitzhugh-Nagumo (FN) [2] equation

$$
u_{t}=u_{x x}+u(1-u)(u-\gamma)
$$

which admits three traveling wave solutions. These solutions describe "shock" type waves connecting the two constant states $u=0$ and $1, u=0$ and $\gamma, u=\gamma$ and 1 ; these waves travel with the particular speeds $\pm \frac{2 \gamma-1}{\sqrt{2}}, \pm \frac{2-\gamma}{\sqrt{2}}, \pm \frac{1+\gamma}{\sqrt{2}}$. These examples should be contrasted with the Burgers' equation

$$
u_{t}=u_{x x}-2 u u_{x}
$$

which admits an exact solution describing a wave traveling with an arbitrary speed.

Burgers' equation is a linearizable equation [3]. Hence, not only it possesses a traveling solution for an arbitrary wave speed, but it also possesses a solution describing the exact interaction of two such waves. It has been assumed that the existence of exact solutions describing two traveling waves is the privilege of linearizable equations only. However, it was shown in [4], that equation (1.1), although not linearizable, admits an exact solution describing the interaction of two of the three waves discussed above ( after interaction, these two waves coalesce and produce the third wave). It was later shown by Satsuma [5] that the situation with the equation

$$
u_{t}=u_{x x}+\alpha u u_{x}+\beta u(1-u)(u-\gamma),
$$

is precisely analogous with that of equation (1.1).

The results of [1] were obtained using the Painlevé property. The results of [4] and [5] were obtained using the bilinear method of Hirota. The two-shock solution found in [4] was also reproduced in [6] using a truncated Painlevé expansion, and in [7] using the non-classical method [8] (or conditional symmetry method [9]-[10]). Some generalizations of these results were presented in [11] using the Painlevé property.

Here we introduce the concept of a generalized conditional symmetry. This concept provides an algorithm for constructing certain exact solutions, such as solutions describing the interaction of two traveling waves. Furthermore, it can be used to construct a large class of evolution equations 
all of which admit the same exact solution. As an example we will show that the FN equation (1.1), and the Satsuma equation (1.3) share the same two-shock solution with the Burgers' equation. More precisely, the equations

$$
\begin{gathered}
u_{t}=u u_{x}-u^{3}+\alpha u+\beta, \\
u_{t}=\frac{1}{3} u_{x x}+\frac{2}{3}\left(-u^{3}+\alpha u+\beta\right), \\
u_{t}=\gamma u_{x x}+(1-3 \gamma) u u_{x}+(1-\gamma)\left(-u^{3}+\alpha u+\beta\right),
\end{gathered}
$$

share the two-shock solution

$$
u=-\left\{\ln \left[K_{1} e^{\alpha_{1} x+\alpha_{1}^{2} t}+K_{2} e^{\alpha_{2} x+\alpha_{2}^{2} t}+K_{3} e^{\alpha_{3} x+\alpha_{3}^{2} t}\right]\right\}_{x},
$$

with the Burgers' equation. In equations (1.4)-(1.7), $K_{1}, K_{2}, K_{3}, \alpha, \beta, \gamma$ are arbitrary constants, and $\alpha_{1}, \alpha_{2}, \alpha_{3}$ are the roots of $x^{3}-\alpha x+\beta=0$, which are assumed to be distinct. It should be noted that because the constants $\alpha$ and $\beta$ appear in equations (1.4)-(1.6), these equations admit a two-traveling wave solution only for certain fixed wave speeds.

As a second example we will show that the equations

$$
u_{t}=u_{x}^{2}+2 u^{2} u_{x}-2 \alpha u_{x}+u^{4}+2 \alpha u^{2}+2 \beta
$$

and

$$
u_{t}=-2 u u_{x x}+2 u_{x}^{2}-2 u^{2} u_{x}-2 \alpha u_{x},
$$

share the same two-soliton solution

$$
u=\left\{\ln \left[K_{1} e^{c_{+} x-\left(2 \alpha-2 c_{-}^{2}\right) c_{+} t}+K_{2} e^{-c_{+} x+\left(2 \alpha-2 c_{-}^{2}\right) c_{+} t}+K_{3} e^{c_{-} x-\left(2 \alpha-2 c_{+}^{2}\right) c_{-} t}+K_{4} e^{-c_{-} x+\left(2 \alpha-2 c_{+}^{2}\right) c_{-} t}\right]\right\}_{x},
$$

with the potential $\mathrm{KdV}$ equation

$$
u_{t}=u_{x x x}+6 u_{x}^{2}
$$

In equations (1.8)-(1.10), $K_{1}, K_{2}, K_{3}, \alpha, \beta$ are arbitrary constants, $c_{ \pm}=\left(-\alpha \pm \sqrt{\alpha^{2}-2 \beta}\right)^{1 / 2}$, and $K_{1} K_{2} c_{+}^{2}=K_{3} K_{4} c_{-}^{2}$. Since $\beta$ does not appear in equation (1.9), this equation admits a one parameter $(\beta)$ family of two-traveling wave solutions. 
We recall that Gel'fand and Dikii [12] extended the concept of a symmetry (or a Lie-point symmetry) by introducing the concept of a generalized (or Lie-Bäcklund [13]) symmetry. Let $K(u$ ) denote a function which depends in a differentiable manner on $u, u_{x}, u_{x x}, \ldots$. The function $\sigma(u)$ is a generalized symmetry of the equation $u_{t}=K(u)$, iff

$$
K^{\prime} \sigma-\sigma^{\prime} K=0
$$

where prime denotes the Fréchet derivative [14]. The concept of a conditional symmetry was introduced in [8] under the name of non-classical symmetry. Generalized conditional symmetries are generalizations of conditional symmetries, in the same way that generalized symmetries are generalizations of symmetries. We introduce the following definition:

Definition 1.1 The function $\sigma(u)$ is a generalized conditional symmetry(GCS) of the equation $u_{t}=K(u)$, iff

$$
K^{\prime} \sigma-\sigma^{\prime} K=F(u, \sigma), \quad F(u, 0)=0,
$$

where $K(u)$ and $\sigma(u)$ are differentiable functions of $u, u_{x}, u_{x x}, \ldots$, while $F(u, \sigma)$ is a differentiable function of $u, u_{x}, u_{x x}, \ldots$ and of $\sigma, \sigma_{x}, \sigma_{x x}, \ldots$

The usefulness of this definition follows from the fact that it implies that the equations $u_{t}=K$ and $\sigma=0$ are compatible. Therefore in general they share a common manifold of solutions. To obtain these solutions one first solves the ODE $\sigma=0$ to obtain $u$ as a function of $x$ with some $x$-independent integration constants. One then substitutes this solution in the equation $u_{t}=K$ to determine the time evolution of these constants. This procedure is precisely the same as the one used for obtaining invariant solutions [15]. It also follows from the definition the following important fact:

Lemma 1.1 If $\sigma$ is a GCS of the equation $u_{t}=K(u)$, and if $G(u, 0)=0$, then $\sigma$ is also a GCS of the equation

$$
u_{t}=K(u)+G(u, \sigma)
$$

Furthermore, all these equations share with equation $u_{t}=K(u)$ the common manifold of solutions obtained through the GCS $\sigma$.

This lemma can be used to explain and generalize the examples discussed earlier. We first discuss equations (1.4)-(1.6). It is well known that the 2-shock solution of the Burgers' equation 
satisfies the third order ODE

$$
\left(u_{x x x}-3 u u_{x x}-3 u_{x}^{2}+3 u^{2} u_{x}\right)+c_{1}\left(u_{x x}-2 u u_{x}\right)+c_{2} u_{x}=0
$$

or

$$
u_{x x}-3 u u_{x}+u^{3}+c_{1}\left(u_{x}-u^{2}\right)+c_{2} u+c_{3}=0 .
$$

The lhs of equation (1.14) is a symmetry of Burgers' equation. It can be verified that the lhs of equation (1.15), which we denote by $\sigma$, is a GCS of Burgers' equation. Therefore, according to the Lemma 1.1, the entire class of equation (1.13), where $K=u_{x x}-2 u u_{x}$ and $\sigma$ is the lhs of equation (1.15), admits the 2-shock solution (1.7). This class contains equation $(1.4)(G(u, \sigma)=$ $-\sigma)$, equation (1.5) $\left(G(u, \sigma)=-\frac{2}{3} \sigma\right)$, and equation $(1.6)(G(u, \sigma)=(\gamma-1) \sigma ;$ in these equations $c_{1}=0, c_{2}=-\alpha$ and $c_{3}=-\beta$ ).

The situation with equations $(1.8),(1.9),(1.11)$ is conceptually similar but technically more interesting. The 2-soliton solution of the potential $\mathrm{KdV}$ equation satisfies the fifth order ODE

$$
u_{x x x x x}+20 u_{x} u_{x x x}+10 u_{x x}^{2}+40 u_{x}^{3}+c_{1}\left(u_{x x x}+6 u_{x}^{2}\right)+c_{2} u_{x}=0
$$

The lhs of equation (1.16) is a symmetry of the potential KdV equation. Actually, equation (1.16) contains the 2-soliton solution as a particular case. The most general solution of equation (1.16) describes the interaction of two-cnoidal waves. It is quite interesting that there exists a second order ODE whose general solution is precisely the 2-soliton solution. This ODE is given by

$$
u_{x x}-\frac{1}{2 u} u_{x}^{2}+2 u u_{x}+\frac{1}{2} u^{3}+\alpha u+\frac{\beta}{u}=0 .
$$

It can be verified that the lhs of equation (1.17), which we denote by $\sigma$, is a GCS of the potential KdV equation. Therefore, according to the Lemma 1.1, the entire class of equation (1.13), where $K=$ $u_{x x x}+6 u_{x}^{2}$ and $\sigma$ is the lhs of equation (1.17), admits the 2-soliton solution (1.10). This class contains equation (1.8) $\left(G(u, \sigma)=K-\sigma_{x}-\left(\frac{u_{x}}{u}+u\right) \sigma\right)$ and equation $(1.9)\left(G(u, \sigma)=K-\sigma_{x}-\left(\frac{u_{x}}{u}+3 u\right) \sigma\right)$.

The above discussion implies that equations (1.4)-(1.6) can be constructed from Burgers' equation and one of its generalized conditional symmetries. Similarly equations (1.8) and (1.9) can be constructed from the potential KdV equation and one of its generalized conditional symmetries. Therefore, the origin of the exact solutions admitted by these non-integrable equations can be traced back to their relationship with integrable equations. In this sense we can think of equations (1.4)-(1.6) and of (1.8)-(1.9) as non-integrable reductions of integrable equations. The 
existence of exact solutions of these non-integrable equations can be thought of as remnants of integrability.

The above results can be generalized to $N$-shock and $N$-soliton solutions. Here we only discuss the $N$-shock case (the $N$-soliton case will be published elsewhere). It is well known that the Burgers' hierarchy $u_{t}=K(u)$, where

$$
K(u)=\sum_{m=0}^{M} \alpha_{m} \partial_{x}\left(\partial_{x}-u\right)^{m} u, \quad M \in \mathcal{Z}^{+},
$$

and $\alpha_{0}, \alpha_{1}, \ldots, \alpha_{M}$ are arbitrary constants, admits an $N$-shock solution. The Lemma 1.1 can be used to obtain the following result:

Theorem 1.1 The class of equation (1.13), where $K(u)$ is given by (1.18) and $\sigma$ is defined by

$$
\sigma=\left(\partial_{x}-u\right)^{N} u+c_{1}\left(\partial_{x}-u\right)^{N-1} u+\cdots+c_{N} u+c_{N+1},
$$

$\left(c_{1}, \ldots, c_{N+1}\right.$ are arbitrary constants), admits the $N$-shock solution

$$
u=-\left\{\ln \left[\sum_{n=1}^{N+1} A_{n} \exp k_{n}\left(x-\left(\sum_{m=0}^{M} \alpha_{m} k_{n}^{m}\right) t\right)\right]\right\}_{x}, \quad M \in \mathcal{Z}^{+}
$$

In equation $(1.20), A_{1}, \ldots, A_{N+1}$ are arbitrary constants and $k_{n}$ are $N+1$ roots of

$$
x^{N+1}+c_{1} x^{N}+\cdots+c_{N} x+c_{N+1}=0 .
$$

A simple consequence of this theorem is that the lowest order PDE, other than a member of Burgers' hierarchy, which admits the $N$-shock solution $(1.20)$ is the $(N-1)$ st order PDE

$$
u_{t}=\sum_{m=0}^{N-1} \alpha_{m} \partial_{x}\left(\partial_{x}-u\right)^{m} u-\alpha_{N-1} \sigma
$$

For example, the equation

$$
u_{t}=u u_{x x}-3 u^{2} u_{x}+u^{4}+\alpha_{1}\left(u_{x x}-2 u u_{x}\right)+\alpha_{0} u_{x}-c_{1}\left(u_{x x}-3 u u_{x}+u^{3}\right)-c_{2}\left(u_{x}-u^{2}\right)-c_{3} u-c_{4},
$$

admits a 3-shock solution.

Details of this theorem are given in $\S 2$. In $\S 3$ we find certain GCS's of the form $\sigma=u_{x x}+g\left(u, u_{x}\right)$ admitted by equations of the form $u_{t}=K\left(u, u_{x}\right)$, where $g$ and $K$ are differentiable functions of $u$ and $u_{x}$. For example we will show that the first order evolution equation

$$
u_{t}=u u_{x}+c_{0} u^{3}+c_{1} u^{2}+c_{2} u+c_{3}
$$


admits the GCS

$$
\sigma=u_{x x}+\left(3 c_{0} u+c_{1}\right) u_{x}+c_{0}\left(c_{0} u^{3}+c_{1} u^{2}+c_{2} u+c_{3}\right) .
$$

We will also show that the first order evolution equation

$$
u_{t}=u_{x}^{2}+\left(c_{1} u^{2}+c_{3}\right) u_{x}+\frac{1}{4} c_{1}^{2} u^{4}+2 c_{4} u^{2}+2 c_{5}-\frac{c_{2}^{2}}{u^{2}}
$$

admits the GCS

$$
\sigma=u_{x x}-\frac{1}{2 u} u_{x}^{2}+\left(c_{1} u+\frac{c_{2}}{u^{2}}\right) u_{x}+\frac{1}{8} c_{1}^{2} u^{3}+c_{4} u+\frac{c_{5}}{u}-\frac{1}{2} \frac{c_{2}^{2}}{u^{3}} .
$$

In $\S 4$ we find the exact solutions corresponding to these GCS's. 


\section{$2 \quad N$-Shock Solutions}

\subsection{The ODE Characterizing the $N$-Shock Solutions}

Lemma 2.1 The $N$-shock solution

$$
u=-\left\{\ln \left[A_{1} e^{k_{1} x}+\cdots+A_{N+1} e^{k_{N+1} x}\right]\right\}_{x}, \quad N \in \mathcal{Z}^{+},
$$

where $A_{1}, \ldots, A_{N+1}$ and $k_{1}, \ldots, k_{N+1}$ are arbitrary constants, satisfies the ODE

$$
\left(\partial_{x}-u\right)^{N} u+c_{1}\left(\partial_{x}-u\right)^{N-1} u+\cdots+c_{N} u+c_{N+1}=0,
$$

where

$$
c_{1}=-\sum_{n=1}^{N+1} k_{n}, \quad c_{2}=\sum_{m<n} k_{m} k_{n}, \cdots, \quad c_{N+1}=(-1)^{N+1} k_{1} \cdots k_{N+1} .
$$

Proof Let $u=-\frac{v_{x}}{v}$, then

$$
\left(\partial_{x}-u\right)^{n} u=\left(\partial_{x}+\frac{v_{x}}{v}\right)^{n} \frac{v_{x}}{v}=\left(\frac{1}{v} \partial_{x} v\right)^{n} \frac{v_{x}}{v}=\frac{1}{v} \partial_{x}^{n+1} v,
$$

equation (2.2) becomes

$$
\partial_{x}^{N+1} v+c_{1} \partial_{x}^{N} v+\cdots+c_{N} v_{x}+c_{N+1} v=0 .
$$

The general solution of this equation is given by

$$
v=A_{1} e^{k_{1} x}+\cdots+A_{N+1} e^{k_{N+1} x},
$$

where $k_{n}$ are $N+1$ roots of $x^{N+1}+c_{1} x^{N}+\cdots+c_{N} x+c_{N+1}=0$.

\subsection{The Burgers' Hierarchy}

Lemma 2.2 The evolution equation defined by

$$
u_{t}=\sum_{m=0}^{M} \alpha_{m} \partial_{x}\left(\partial_{x}-u\right)^{m} u, \quad M \in \mathcal{Z}^{+},
$$

where $\alpha_{0}, \ldots, \alpha_{M}$ are arbitrary constants, admits the $N$-shock solution

$$
u=-\left\{\ln \left[\sum_{n=1}^{N+1} A_{n} \exp k_{n}\left(x-\left(\sum_{m=0}^{M} \alpha_{m} k_{n}^{m}\right) t\right)\right]\right\}_{x}, \quad M \in \mathcal{Z}^{+},
$$

where $A_{1}, \ldots, A_{N+1}$ and $k_{1}, \ldots, k_{N+1}$ are arbitrary constants.

Proof Let $u=-\frac{v_{x}}{v}$, then equation (2.3) becomes

$$
v_{t}+\sum_{m=0}^{M} \alpha_{m} \partial_{x}^{m+1} v=0
$$


which admits the solution

$$
v=\exp \left(k_{n} x-\left(\sum_{m=0}^{M} \alpha_{m} k_{n}^{m+1}\right) t\right),
$$

as well as a linear combinations of such solutions.

Remark Equation (2.3) with $m=1, \alpha_{0}=0, \alpha_{1}=1$ is Burgers' equation. It is known that the Burgers' hierarchy is given by $u_{t}=\sum_{m=0}^{M} \Phi^{m} u_{x}$, where $\Phi=\partial_{x}-u-u_{x} \partial_{x}^{-1}$. Using induction it is easy to show that $\Phi^{m} \partial_{x}=\partial_{x}\left(\partial_{x}-u\right)^{m}$, which implies equation (2.3).

2.3 Generalized Conditional Symmetries of the Burgers' Hierarchy Associated with the $N$-Shock Solutions

The $N$-shock solution (2.4) is the common solution of the ODE (2.2) and the evolution equation (2.3). Thus we expect that the lhs of equation (2.2) is a GCS of equation (2.3). This in indeed the case.

Lemma 2.3 The evolution equation (2.3) has the generalized conditional symmetry $\sigma$, where $\sigma$ is the lhs of equation (2.2).

Proof We need only to verify that $\sigma$ is a GCS of the equation

$$
u_{t}=\partial_{x}\left(\partial_{x}-u\right)^{m} u
$$

Let $c_{0}=1$, then

$$
\begin{gathered}
\sigma=\sum_{n=0}^{N} c_{N-n}\left(\partial_{x}-u\right)^{n} u+c_{N+1} \\
\sigma^{\prime}\left[\partial_{x}\left(\partial_{x}-u\right)^{m} u\right]=\sum_{n=0}^{N} c_{N-n}\left\{\left(\partial_{x}-u\right)^{m+n+1} u+\left[\left(\partial_{x}-u\right)^{m} u\right]\left[\left(\partial_{x}-u\right)^{n} u\right]\right\} \\
=\left(\partial_{x}-u\right)^{m+1}\left[\sum_{n=0}^{N} c_{N-n}\left(\partial_{x}-u\right)^{n} u\right]+\left[\left(\partial_{x}-u\right)^{m} u\right]\left[\sum_{n=0}^{N} c_{N-n}\left(\partial_{x}-u\right)^{n} u\right] \\
=\left(\partial_{x}-u\right)^{m+1} \sigma+\left[\left(\partial_{x}-u\right)^{m} u\right] \sigma
\end{gathered}
$$

which vanishes when $\sigma=0$.

\subsection{Evolution Equations Admitting An $N$-Shock Solution}

The Theorem 1.1 presented in the introduction follows from Lemmas 1.1, 2.1-2.3.

The $N$-shock solution satisfies the $N$ st order ODE $\sigma=0$, where $\sigma$ is the lhs of equation (2.2). On the other hand, the equation (2.3) with $M=N-1$ admits $\sigma=0$. Hence the equation (1.22) admits the $N$-shock solution (1.20).

Example 1 The equation

$$
u_{t}=u^{2}-c_{1} u-c_{2}
$$


admits a 1-shock solution.

Example 2 The equation

$$
u_{t}=u u_{x}-u^{3}+\alpha u_{x}-c_{1}\left(u_{x}-u^{2}\right)-c_{2} u-c_{3}
$$

admits a 2-shock solution. 


\section{Derivation of Some Generalized Conditional Symmetries}

The definition (1.12) implies that the equation $u_{t}=K\left(u, u_{x}\right)$ admits the GCS $\sigma=u_{x x}+g\left(u, u_{x}\right)$ iff

$$
\left.\sigma^{\prime} K\right|_{\sigma=0}=K_{00} u_{x}^{2}-2 g K_{01} u_{x}+g^{2} K_{11}+\left(g_{1} K_{0}-g_{0} K_{1}\right) u_{x}+\left(g_{0} K-g K_{0}\right)=0
$$

In eq.(3.1) $K$ and $g$ are differentiable functions of $u$ and $u_{x}$ and the subscripts 0 and 1 denote differentiation with respect to $u$ and $u_{x}$.

We first discuss the case that $K$ is linear in $u_{x}$. We will show that the equation

$$
u_{t}=S(u) u_{x}+T(u)
$$

admits the GCS

$$
\sigma=u_{x x}-\frac{S^{\prime}}{T} u_{x}^{3}-\frac{T^{\prime}}{T} u_{x}^{2}+\sum \frac{c_{n} u_{x}^{n}}{T^{2}\left(S u_{x}+T\right)^{n-3}}
$$

In equation (3.3), S(u) and $T(u)$ are arbitrary functions of $u, c_{n}$ are arbitrary constants and the summation is over all integers. The derivation is as follows. Let

$$
g\left(u, u_{x}\right)=\sum f_{n}(u) u_{x}^{n}
$$

then equation (3.1) yields

$$
\delta_{n, 3} S^{\prime \prime}+\delta_{n, 2} T^{\prime \prime}+(n-4) f_{n-1} S^{\prime}+(n-1) f_{n} T^{\prime}+f_{n}^{\prime} T=0
$$

Thus

$$
f_{n}=\frac{-\delta_{n, 3} S^{\prime} T-\delta_{n, 2} T^{\prime}+\sum_{k=0}^{n-4}(-1)^{k}\left(\begin{array}{c}
n-4 \\
k
\end{array}\right) c_{n-k}}{T^{n-1}}
$$

and equation (3.3) follows.

In the particular case that $S(u)=u, T(u)$ is a cubic polynomial and $c_{n}=0(n \geq 4$ or $<0)$, equations (3.2) and (3.3) becomes equations (1.22) and (1.23).

We now discuss the case that $K$ is quadratic in $u_{x}$. Using the definition (1.12), it is also possible to solve the following problem: Find all first order equations of the form

$$
u_{t}=R(u) u_{x}^{2}+S(u) u_{x}+T(u)
$$

which admit a GCS of the form 


$$
\sigma=d(u) u_{x}^{2}+f(u) u_{x}+h(u)
$$

In equations (3.4) and (3.5), $R(u), S(u), T(u), d(u), f(u), h(u)$ are differentiable functions of $u$. It turns out that, within a change of variables of the form $u=V(v)$, there exist the following four cases:

(i) The equation

$$
u_{t}=u_{x}^{2}+\left(c_{1} u^{2}+c_{3}\right) u_{x}+\frac{1}{4} c_{1}^{2} u^{4}+2 c_{4} u^{2}+2 c_{5}-\frac{c_{2}^{2}}{u^{2}}
$$

admits the GCS

$$
\sigma=u_{x x}-\frac{1}{2 u} u_{x}^{2}+\left(c_{1} u+\frac{c_{2}}{u^{2}}\right) u_{x}+\frac{1}{8} c_{1}^{2} u^{3}+c_{4} u+\frac{c_{5}}{u}-\frac{1}{2} \frac{c_{2}^{2}}{u^{3}} .
$$

(ii) The equation

$$
u_{t}=u_{x}^{2}+\left(4 c_{1} u+2 c_{2} \sqrt{u}+c_{3}\right) u_{x}+4 c_{1}^{2} u^{2}+4 c_{1} c_{2} u \sqrt{u}+2 c_{4} u+2 c_{5} \sqrt{u}
$$

admits the GCS

$$
\sigma=u_{x x}+\left(3 c_{1}+\frac{c_{2}}{\sqrt{u}}\right) u_{x}+2 c_{1}^{2} u+2 c_{1} c_{2} \sqrt{u}+c_{4}+\frac{c_{5}}{\sqrt{u}}
$$

(iii) The equation

$$
u_{t}=c_{1} u\left(u_{x}+a u\right)^{2}+c_{2}\left(u_{x}+a u\right)^{2}+c_{3} u\left(u_{x}+a u\right)+c_{4}\left(u_{x}+a u\right)+c_{5} u+c_{6},
$$

admits the GCS

$$
\sigma=u_{x x}+a u_{x}
$$

(iv) The equation

$$
u_{t}=u_{x}^{2}+S(u) u_{x}
$$

admits the GCS

$$
\sigma=u_{x x}+S^{\prime}(u) u_{x} .
$$


The derivation of the above result is tedious but straightforward. We shall only indicate how the case (i) can be obtained. Suppose that $K$ and $g$ are polynomials in $u_{x}$ of order $m>1$ and $n$, respectively. Then the order of $g^{2} K_{11}$ is $m+2 n-1$, while the orders of the other terms in equation (3.1) are less or equal to $m+2$ or $m+n$. Thus $n \leq 2$, i.e., $\sigma$ is of the form (3.5). Hence even if $K$ is an arbitrary polynomial of $u_{x}$ of order $m>1, g$ is only a second order polynomial in $u_{x}$. In the particular case that $K$ is a second order polynomial in $u_{x}$, equation (3.1) yields

$$
\begin{gathered}
R^{\prime \prime}-3 d R^{\prime}-d^{\prime} R+2 d^{2} R=0, \quad S^{\prime \prime}-d S^{\prime}-4 f R^{\prime}-f^{\prime} R+4 d f R=0, \\
T^{\prime \prime}+d T^{\prime}+d^{\prime} T-2 f S^{\prime}-5 h R^{\prime}-h^{\prime} R+4 d h R+2 f^{2} R=0, \\
-3 h S^{\prime}+f^{\prime} T+4 f h R=0, \quad 2 h^{2} R+h^{\prime} T-h T^{\prime}=0 .
\end{gathered}
$$

Suppose that $h \neq 0$, then equation $(3.14 \mathrm{e})$ yields

$$
R=r^{\prime}, \quad T=2 h r
$$

Equation (3.14a) is a Riccati equation for $d$. Its integration yields

$$
d=-\frac{1}{2}\left[\ln \left(\frac{a_{1} r+a_{2}}{\left(r^{\prime}\right)^{2}}\right)\right]^{\prime}
$$

Here we consider the case that $a_{2}=0$, then

$$
d=\frac{r^{\prime \prime}}{r^{\prime}}-\frac{1}{2} \frac{r^{\prime}}{r}
$$

From equation (3.14d), we find

$$
S^{\prime}=\frac{2}{3} f^{\prime} r+\frac{4}{3} f r^{\prime}
$$

Substitute this into equation(3.14b) we obtain

$$
f=c_{1} r+\frac{c_{2}}{r^{2}}, \quad S=c_{1} r^{2}+c_{3}
$$

Then equation(3.14c) yields

$$
\begin{gathered}
h^{\prime}+h \frac{r^{\prime}}{r}+h \frac{r^{\prime \prime}}{r^{\prime}}-\frac{1}{2} c_{1}^{2} r^{2}-\frac{1}{4} \frac{c_{2}^{2}}{r^{4}}=2 c_{4}, \\
h=\frac{1}{8} \frac{c_{1}^{2} r^{3}}{r^{\prime}}-\frac{1}{2} \frac{c_{2}^{2}}{r^{3} r^{\prime}}+\frac{c_{4} r}{r^{\prime}}+\frac{c_{5}}{r r^{\prime}} .
\end{gathered}
$$


Thus we find that the evolution equation

$$
u_{t}=r^{\prime} u_{x}^{2}+\left(c_{1} r^{2}+c_{3}\right) u_{x}+\frac{2 r}{r^{\prime}}\left(\frac{1}{8} c_{1}^{2} r^{3}+c_{4} r+\frac{c_{5}}{r}-\frac{1}{2} \frac{c_{2}^{2}}{r^{3}}\right)
$$

admits the GCS

$$
\sigma=u_{x x}+\left(\frac{r^{\prime \prime}}{r^{\prime}}-\frac{r^{\prime}}{2 r}\right) u_{x}^{2}+\left(c_{1} r+\frac{c_{2}}{r^{2}}\right) u_{x}+\frac{1}{8} \frac{c_{1}^{2} r^{3}}{r^{\prime}}+\frac{c_{4} r}{r^{\prime}}+\frac{c_{5}}{r r^{\prime}}-\frac{1}{2} \frac{c_{2}^{2}}{r^{3} r^{\prime}},
$$

where $r=r(u)$ is an arbitrary function and $c_{1}, c_{2}, c_{3}, c_{4}, c_{5}$ are arbitrary constants. This equation is equivalent to a special case $r(u)=u$, i.e., to equation $(3.6)$. 


\section{Derivation of Some Particular Solutions}

We first consider equation (1.22) and the GCS (1.23).

(a) $c_{0}=c_{1}=0$. The equation

$$
u_{t}=u u_{x}+c_{2} u+c_{3}
$$

admits the GCS

$$
\sigma=u_{x x}
$$

The equation $\sigma=0$ implies $u=k_{1}(t) x+k_{2}(t)$, then equation (3.1) yields $k_{1}^{\prime}=k_{1}^{2}+c_{2} k_{1}, k_{2}^{\prime}=$ $k_{1} k_{2}+c_{2} k_{2}+c_{3}$. The associated solutions for $c_{2}=0$ and $c_{2}=1$ are

$$
u=\frac{K_{1}\left(-x+\frac{1}{2} c_{3} t^{2}\right)+K_{2} c_{3} t+K_{3}}{K_{1} t+K_{2}} \text { and } u=\frac{\left(-K_{1} x+c_{3} K_{1} t+K_{3}\right) e^{t}-c_{3} K_{2}}{K_{1} e^{t}+K_{2}}
$$

respectively.

(b) $c_{0}=0, c_{1}=1$. The equation

$$
u_{t}=u u_{x}+u^{2}+c_{2} u+c_{3}
$$

admits the GCS

$$
\sigma=u_{x x}+u_{x}
$$

The associated solutions are

$$
u=\frac{-K_{1}\left(\frac{1}{2} c_{2} t+1\right)-\frac{1}{2} K_{2} c_{2}+K_{3} e^{-x+\frac{1}{2} c_{2} t}}{K_{1} t+K_{2}}, \text { for } c_{3}=-\frac{1}{4} c_{2}^{2}
$$

and

$$
u=\frac{-K_{1} c_{+} e^{c_{+} t}-K_{2} c_{-} e^{c_{-} t}+K_{3} e^{-x+c_{2} t}}{K_{1} e^{c_{+} t}+K_{2} e^{c_{-} t}}, \text { for } c_{3} \neq-\frac{1}{4} c_{2}^{2}
$$

where $c_{ \pm}=\frac{1}{2}\left(c_{2} \pm \sqrt{c_{2}^{2}+4 c_{3}}\right)$.

(c) $c_{0}=-1$. The equation

$$
u_{t}=u u_{x}-u^{3}+c_{1} u^{2}+c_{2} u+c_{3}
$$

admits the GCS 


$$
\sigma=u_{x x}+\left(-3 u+c_{1}\right) u_{x}+u^{3}-c_{1} u^{2}-c_{2} u-c_{3} .
$$

Equation $\sigma=0$ is Painlevé type equation number 6 in Gambier's classification [16]. Let $u=-\frac{v_{x}}{v}$, then

$$
v_{x x x}+c_{1} v_{x x}-c_{2} v_{x}+c_{3} v=0, \quad v_{t}=v_{x x}+c_{1} v_{x}
$$

Let $a_{1}, a_{2}$ and $a_{3}$ be the three roots of the cubic equation $a^{3}+c_{1} a^{2}-c_{2} a+c_{3}=0$, then the associated solution is

$$
\begin{gathered}
u=-\left\{\ln \left[K_{1} e^{a_{1} x+\left(a_{1}^{2}+a_{1} c_{1}\right) t}+K_{2} e^{a_{2} x+\left(a_{2}^{2}+a_{2} c_{1}\right) t}+K_{3} e^{a_{3} x+\left(a_{3}^{2}+a_{3} c_{1}\right) t}\right]\right\}_{x}, \text { for } a_{1} \neq a_{2} \neq a_{3} ; \\
u=-\left\{\ln \left[\left(K_{1}\left(x+\left(2 a_{1}+c_{1}\right) t\right)+K_{2}\right) e^{a_{1} x+\left(a_{1}^{2}+a_{1} c_{1}\right) t}+K_{3} e^{a_{3} x+\left(a_{3}^{2}+a_{3} c_{1}\right) t}\right]\right\}_{x}, \text { for } a_{1}=a_{2} \neq a_{3} ;
\end{gathered}
$$

and

$$
u=\frac{1}{3} c_{1}-\frac{2 K_{1}\left(x+\frac{1}{3} c_{1} t\right)+K_{2}}{K_{1}\left[\left(x+\frac{1}{3} c_{1} t\right)^{2}+2 t\right]+K_{2}\left(x+\frac{1}{3} c_{1} t\right)+K_{3}}, \text { for } a_{1}=a_{2}=a_{3}
$$

We next consider the four cases (i)-(iv).

Case (i).

(a) $c_{1}=2, c_{2}=0$. The equation

$$
u_{t}=u_{x}^{2}+2 u^{2} u_{x}+c_{3} u_{x}+u^{4}+2 c_{4} u^{2}+2 c_{5}
$$

admits the GCS

$$
\sigma=u_{x x}-\frac{1}{2 u} u_{x}^{2}+2 u u_{x}+\frac{1}{2} u^{3}+c_{4} u+\frac{c_{5}}{u} .
$$

Equation $\sigma=0$ is Painlevé type equation number 27 in Gambier's classification [16]. Let $u=\frac{v_{x}}{v}$, then

$$
v_{x} v_{x x x}-\frac{1}{2} v_{x x}^{2}+c_{4} v_{x}^{2}+c_{5} v^{2}=0, \quad\left(\frac{v_{t}}{v}\right)_{x}=\frac{v_{x x}^{2}}{v^{2}}+c_{3}\left(\frac{v_{x}}{v}\right)_{x}+2 c_{4} \frac{v_{x}^{2}}{v^{2}}+2 c_{5} .
$$

It turns out that $v$ also satisfies the following linear equations

$$
v_{x x x x}+2 c_{4} v_{x x}+2 c_{5} v=0, \quad v_{t}+2 v_{x x x}+\left(-c_{3}+4 c_{4}\right) v_{x}=0
$$

There exist the following subcases:

1. $c_{4}=c_{5}=0$. Then 


$$
u=\frac{3 K_{1}\left(K_{1} x+c_{3} K_{1} t+K_{2}\right)^{2}}{\left(K_{1} x+c_{3} K_{1} t+K_{2}\right)^{3}-12 K_{1}^{3} t+K_{3}}, \quad \text { and } \quad u=\frac{K_{1}}{K_{1} x+c_{3} K_{1} t+K_{2}} .
$$

2. $c_{4} \neq 0, c_{5}=0$. Then

$$
u=\left\{\ln \left[K_{1} e^{c x+\left(c c_{3}+c^{3}\right) t}+K_{2} e^{-c x-\left(c c_{3}+c^{3}\right) t} \pm 2 c \sqrt{-K_{1} K_{2}}\left(x+\left(c_{3}+2 c^{2}\right) t\right)+K_{3}\right]\right\}_{x}, \quad c=\sqrt{-2 c_{4}} .
$$

3. $c_{5}=\frac{1}{2} c_{4}^{2} \neq 0$. Then

$u=\left\{\ln \left[\left(K_{1} x+\left(c_{3}-2 c^{2}\right) K_{1} t+K_{2}\right) e^{c x+\left(c c_{3}+2 c^{3}\right) t}+\left(K_{3} x+\left(c_{3}-2 c^{2}\right) K_{3} t+K_{4}\right) e^{-c x-\left(c c_{3}+2 c^{3}\right) t}\right]\right\}_{x}, \quad c=\sqrt{-c_{4}}$,

where

$$
2 K_{1} K_{3}+c\left(K_{2} K_{3}-K_{1} K_{4}\right)=0
$$

4. $c_{5} \neq \frac{1}{2} c_{4}^{2}, 0$. Then

$$
u=\left\{\ln \left[K_{1} e^{c_{+} x+\left(c_{3}+2 c_{-}^{2}\right) c_{+} t}+K_{2} e^{-c_{+} x-\left(c_{3}+2 c_{-}^{2}\right) c_{+} t}+K_{3} e^{c_{-} x+\left(c_{3}+2 c_{+}^{2}\right) c_{-} t}+K_{4} e^{-c_{-} x-\left(c_{3}+2 c_{+}^{2}\right) c_{-} t}\right]\right\}_{x},
$$

where $c_{ \pm}=\sqrt{-c_{4} \pm \sqrt{c_{4}^{2}-2 c_{5}}}$ and $K_{1} K_{2} c_{+}^{2}=K_{3} K_{4} c_{-}^{2}$.

(b) $c_{1}=c_{2}=0$. The equation

$$
u_{t}=u_{x}^{2}+c_{3} u_{x}+2 c_{4} u^{2}+2 c_{5}
$$

admits the GCS

$$
\sigma=u_{x x}-\frac{1}{2 u} u_{x}^{2}+c_{4} u+\frac{c_{5}}{u}
$$

The equation $\sigma=0$ implies that $u$ also satisfies the linear equation $u_{x x x}+2 c_{4} u_{x}=0$. There exist the following subcases:

1. $c_{4}=0$. Then

$$
u=-\frac{\left(x+c_{3} t+K_{2}\right)^{2}}{4\left(t+K_{1}\right)}+2 c_{5}\left(t+K_{1}\right), \text { and } u= \pm \sqrt{2 c_{5}}\left(x+c_{3} t\right)+4 c_{5} t+K
$$

2. $c_{4}=-\frac{1}{2}$. Then

$$
u=\frac{\sqrt{2 c_{5}}\left(K_{1} e^{2 \sqrt{2 c_{5}} t}-K_{2} e^{-2 \sqrt{2 c_{5}} t}\right)+K_{3} e^{x+c_{3} t}+K_{4} e^{-x-c_{3} t}}{K_{1} e^{2 \sqrt{2 c_{5}} t}+K_{2} e^{-2 \sqrt{2 c_{5}} t}}
$$

where

$$
2 c_{5} K_{1} K_{2}+K_{3} K_{4}=0
$$


Case (ii).

(a) $c_{1}=c_{2}=c_{5}=0$. The equation

$$
u_{t}=u_{x}^{2}+c_{3} u_{x}+2 c_{4} u
$$

admits the GCS

$$
\sigma=u_{x x}+c_{4}
$$

The associated solutions are

$$
u=K_{1} x+\left(K_{1}^{2}+c_{3} K_{1}\right) t+K_{2}, \quad \text { for } c_{4}=0
$$

and

$$
u=-\frac{1}{2} c_{4} x^{2}+\left(-c_{3} c_{4} t+K_{1}\right) x-\frac{1}{2} c_{3}^{2} c_{4} t^{2}+c_{3} K_{1} t-\frac{K_{1}^{2}}{2 c_{4}}+K_{2} e^{2 c_{4} t}, \quad \text { for } \quad c_{4} \neq 0
$$

(b) $c_{1}=1, c_{2}=c_{5}=0$. The equation

$$
u_{t}=u_{x}^{2}+4 u u_{x}+c_{3} u_{x}+4 u^{2}+2 c_{4} u
$$

admits the GCS

$$
\sigma=u_{x x}+3 u_{x}+2 u+c_{4}
$$

The associated solutions are

$$
u=K_{1} e^{-x-c_{3} t}+\left(K_{1}^{2} t+K_{2}\right) e^{-2 x-2 c_{3} t}, \text { for } c_{4}=0
$$

and

$$
u=K_{1} e^{-x-c_{3} t}-\frac{K_{1}^{2}}{2 c_{4}} e^{-2 x-2 c_{3} t}+K_{2} e^{-2 x+2\left(c_{4}-c_{3}\right) t}-\frac{1}{2} c_{4}, \text { for } \quad c_{4} \neq 0 .
$$

(c) $c_{1}=c_{3}=c_{4}=c_{5}=0, c_{2}=-1$. The equation

$$
u_{t}=u_{x}^{2}-2 \sqrt{u} u_{x}
$$

admits the GCS

$$
\sigma=u_{x x}-\frac{u_{x}}{\sqrt{u}}
$$


It is more convenient to study the equivalent equation

$$
u_{t}=2 u u_{x}^{2}-2 u u_{x}
$$

which admits the GCS

$$
\sigma=u_{x x}+\frac{u_{x}^{2}}{u}-\frac{u_{x}}{u}
$$

The associated solution is defined by

$$
\left(u+K_{1}\right)^{K_{1}}=K_{2} e^{u-x-2 K_{1} t}
$$

Case (iii).

(a) $a=0$. The associated solution is

$$
u=k(t)\left[x+\int\left(c_{2} k(t)+c_{4}+\frac{c_{6}}{k(t)}\right) d t\right]
$$

where $k(t)$ satisfies

$$
k^{\prime}=c_{1} k^{3}+c_{3} k^{2}+c_{5} k
$$

(b) $a=-1$. The associated solution is

$$
u=k(t)+e^{x+\int\left(c_{1} k^{2}(t)-c_{3} k(t)+c_{5}\right) d t}
$$

where $k(t)$ satisfies

$$
k^{\prime}=c_{1} k^{3}+\left(c_{2}-c_{3}\right) k^{2}+\left(-c_{4}+c_{5}\right) k+c_{6}
$$

Case (iv).

The associated solution is defined by

$$
\int \frac{d u}{K-S(u)}=x+K t
$$

For example, with $S(u)=u$, we find that the equation

$$
u_{t}=u_{x}^{2}+u u_{x}
$$

admits the GCS $\sigma=u_{x x}+u_{x}$, and the solution

$$
u=K_{1}+K_{2} e^{-\left(x+K_{1} t\right)} .
$$


Also with $S(u)=u^{2}$, we find that the equation

$$
u_{t}=u_{x}^{2}+u^{2} u_{x}
$$

admits the GCS $\sigma=u_{x x}+2 u u_{x}$, and the solutions

$$
u=\frac{1}{x+K} \text { and } u=\frac{K_{1}\left(K_{2} e^{2 K_{1}\left(x+K_{1}^{2} t\right)}-K_{3}\right)}{K_{2} e^{2 K_{1}\left(x+K_{1}^{2} t\right)}+K_{3}}
$$

\section{Remarks}

We conclude with some remarks.

(a) It is possible to obtain many more evolution equations and their generalized conditional symmetries by assuming that $K\left(u, u_{x}\right)$ is a polynomial in $u_{x}$ of order $\geq 3$. For example, it is easy to verify that the evolution equation

$$
u_{t}=\frac{1}{u^{m-2}}\left(u_{x}+u^{2}\right)^{m}, \quad m \in \mathcal{Z}^{+}
$$

admits the GCS

$$
\sigma=u_{x x}-\frac{m-1}{m} \frac{u_{x}^{2}}{u}+\frac{m+2}{m} u u_{x}+\frac{1}{m} u^{3} .
$$

Equation $\sigma=0$ is Painlevé type equation number 24 in Gambier's classification [16].

(b) A conditional symmetry of the equation $u_{t}=K$, is the particular case of a GCS given by

$$
\sigma=a(u) u_{x}+b(u) K-c(u)
$$

(In this case the associated vector field is $a(u) \frac{\partial}{\partial x}+b(u) \frac{\partial}{\partial t}+c(u) \frac{\partial}{\partial u}[17]$ ).

Recall that the case of two-shock solution corresponds to a GCS of the form $\sigma=u_{x x}+g\left(u, u_{x}\right)$. This $\sigma$ is a GCS of the first order PDE $u_{t}=K\left(u, u_{x}\right)$. However, for some of the second order PDE's $u_{t}=u_{x x}+\tilde{K}\left(u, u_{x}\right), \sigma$ can be written in the form (4.3). This is the case, for example, for equation (1.5). This is why the two-shock wave solution of this equation was also obtained by using conditional symmetries [7] as opposed to GCS.

(c) A given evolution equation may admit more than one GCS of a given order. For example the Burgers' equation, in addition to the GCS given by the lhs of equation (1.15), also admits the following GCS's

$$
\sigma_{1}=u_{x x}, \quad \sigma_{2}=u_{x x}-\frac{1}{u} u_{x}^{2}+\left(-u+\frac{c}{u}\right) u_{x}
$$


Also the potential KdV equation, in addition to the GCS given by the lhs of equation (1.17), also admits the following GCS's

$$
\sigma_{1}=u_{x x}, \quad \sigma_{2}=u_{x x}-\frac{1}{2 u} u_{x}^{2}, \quad \sigma_{3}=u_{x x}+2 u u_{x}+c u_{x}, \quad \sigma_{4}=u_{x x}-\frac{1}{u} u_{x}^{2}+\left(u+\frac{c}{u}\right) u_{x}
$$

\section{Acknowledgements}

This work was partially supported by the National Science Foundation under Grant Number DMS-9204075, and by the Air Force Office of Scientific Research under the Grant Number F4962093-1-0088-DEP. 


\section{Reference}

1. M.J.Ablowitz and A.Zeppetella, Bull.Math.Biol. 41(1979) 835.

2. R.Fitzhugh, Biophys J. 1(1961) 445; J.S.Nagumo, S.Arimoto and S.Yoshizawa, Proc.IRE 50(1962) 2061; A.Kolmogorov, I.Petrovsky and N.Piscounov, Bull.Univ.Etat.Moscou (Ser.Int.) A1(1937) 1; A.C.Newell and J.A.Whitehead, J.Fluid Mech. 38(1969) 239.

3. J.D.Cole, Quart.Appl.Math. 9(1951) 225; E.Hopf, Comm.Pure Appl.Math. 3(1950) 201.

4. T.Kawahara and M.Tanaka, Phys.Lett. 97A(1983) 311.

5. J.Satsuma, Exact solutions of Burgers' equation with reaction terms, preprint, 1987.

6. R.Conte, Phys.Lett.134A(1988) 100; F.Cariello and M.Tabor, Physica 39D(1989) 77; P.G.Estévez and P.R.Gordoa, J.Phys.23A(1990) 4831; Z.Chen and B.Guo, IMA J.Appl.Math. 48(1992) 645.

7. M.C.Nucci and P.A.Clarkson, Phys.Lett. 164A(1992) 49-56.

8. G.W.Bluman and J.D.Cole, J.Math.Mech., 18(1969) 1025.

9. P.J.Olver and P.Rosenau, Phys.Lett. 114A(1986) 107; SIAM J.Appl.Math. 47(1987) 263.

10. P.A.Clarkson and P.Winternitz, Physica D 49(1991) 257.

11. N.A.Kudryashov, Phys.Lett. 169A(1992) 237.

12. I.M.Gel'fand and L.A.Dikii, Uspeki Matem Nauk 30(1975) 67.

13. R.L.Anderson and N.H.Ibragimov, Lie-Bäcklund Transformations in Applications, SIAM Stud. Appl.Math. No.1, Philadelphia, 1979.

14. A.S.Fokas and I.M.Gel'fand, Bi-hamiltonian structures and integrability, in "Important Devel- 
opment in Soliton Theory", ed. by A.S.Fokas and V.E.Zakharov, Springer Verlag, 1993.

15. L.V.Ovsiannikov, Group Analysis of Differential Equations, Academic, New York, 1982.

16. E.L.Ince, Ordinary Differential Equations, Longmans \& Green, London and New York, 1926.

17. A.S.Fokas, J.Math.Phys. 21(1980) 1318; Stud.Appl.Math. 77(1987) 253. 
